\title{
Nonlinear crystals for imaging and detection of mid-IR radiation
}

\author{
Pedersen, C.; Ashik, A. S.; Tidemand-Lichtenberg, P.
}

Published in:

Proceedings of SPIE

Link to article, DOI:

$10.1117 / 12.2550844$

Publication date:

2020

Document Version

Publisher's PDF, also known as Version of record

Link back to DTU Orbit

Citation (APA):

Pedersen, C., Ashik, A. S., \& Tidemand-Lichtenberg, P. (2020). Nonlinear crystals for imaging and detection of mid-IR radiation. In S. He, S. He, \& L. Vivien (Eds.), Proceedings of SPIE (Vol. 112841). [112841A] SPIE International Society for Optical Engineering. Proceedings of SPIE - The International Society for Optical Engineering Vol. 11284 https://doi.org/10.1117/12.2550844

\section{General rights}

Copyright and moral rights for the publications made accessible in the public portal are retained by the authors and/or other copyright owners and it is a condition of accessing publications that users recognise and abide by the legal requirements associated with these rights.

- Users may download and print one copy of any publication from the public portal for the purpose of private study or research.

- You may not further distribute the material or use it for any profit-making activity or commercial gain

- You may freely distribute the URL identifying the publication in the public portal 


\section{Nonlinear crystals for imaging and detection of mid-IR radiation}

Pedersen, C., A. S., Ashik, Tidemand-Lichtenberg, P.

C. Pedersen, Ashik A. S., P. Tidemand-Lichtenberg, "Nonlinear crystals for imaging and detection of mid-IR radiation," Proc. SPIE 11284, Smart Photonic and Optoelectronic Integrated Circuits XXII, 112841A (26 February 2020); doi: $10.1117 / 12.2550844$

SPIE. Event: SPIE OPTO, 2020, San Francisco, California, United States 


\title{
Nonlinear crystals for imaging and detection of mid-IR radiation \\ C. Pedersen*a, Ashik. A.S. ${ }^{a}$, P. Tidemand-Lichtenberg ${ }^{\mathrm{a}}$ \\ ${ }^{a}$ DTU Fotonik, Department of Photonics Engineering, Building 343, Ørsteds Plads, 2800 Lyngby, Denmark
}

\begin{abstract}
The mid-IR wavelength range, i.e. 2-25 $\mu \mathrm{m}$, houses the vibrational spectra of most gasses and large molecules found in complex structures such as tissue, plastics or food. When designing an upconversion system for imaging or mid-IR detection, several aspects should be considered. Upconversion has several advantageous features which makes it an interesting option to consider for many mid-IR applications. Main advantages include, low-noise detection even at elevated temperatures, an almost instantaneous response time, phase preservation, and the ability to perform upconversion imaging. The $\chi^{(2)}$ crystal properties impact the performance of the system significantly. Design aspects related to the choice of crystal as well as different applications is presented.
\end{abstract}

Keywords: Upconversion imaging, mid-IR imaging, nonlinear phase matching, sum frequency generation

\section{INTRODUCTION}

The mid-infrared (mid-IR) wavelength range $(2-25 \mu \mathrm{m})$ is an emerging new topic for frontier research. Its general importance relates to a multitude of mid-IR industrial and biomedical sensor applications, which can be addressed via vibration spectroscopy ${ }^{1}$. The "chemical finger prints" of most complex molecules such as those found in food, tissue or catalytic compounds all have vibrational spectra in the mid-IR ${ }^{1}$. Likewise, fundamental absorption bands of many gas molecules, i.e. where gas molecules have their strongest absorption and emission of light, are also centred in the 2-13 $\mu \mathrm{m}$ range. Improved detectors in this wavelength range therefor enables novel instrumentation for mid-IR gas spectroscopy at small concentrations, relevant for applications like, "leak-finding" or remote sensing of greenhouse gases ${ }^{2}$.

One important obstacle for the exploitation of the mid-IR optical region has been the lack of sensitive mid-IR detectors and imaging devices. Here we present a unique mid-IR upconversion detection and imaging technique as an attractive alternative to cryogenic cooled detectors. Upconversion has inherently low noise, approaching single photon detection, even using room temperature devices. The time response in the conversion process appear "instantaneous" for most practical applications, relying on the $\chi^{(2)}$ electronic response of the nonlinear crystal, hence limited by the detection system, i.e. the NIR detector response.

Upconversion imaging shares many properties with standard linear imaging, as well-known from text books, however two aspects differ significantly. Firstly, the $\chi^{(2)}$ interaction is inherently much weaker than its linear counterpart, thus imposing design constraints in order to optimise the efficiency. Secondly, when imaging is wanted, the phase mismatch properties requires special attention in order to obtain a large Field-of-View (FoV). Both of these aspects depend strongly on the choice of nonlinear crystal. In this paper we will present (crystal) design aspects, affecting the performance of upconversion detection and imaging, ranging from $\mathrm{CW}$ to the fs regime.

\section{CRYSTAL SELECTION}

\subsection{Transparency range}

A first selection criterion is the transparency range of the nonlinear material. Three wave mixing requires the interaction of three different wavelengths namely signal, pump and upconverted wavelengths. Fig.1. shows the general case where a

Smart Photonic and Optoelectronic Integrated Circuits XXII, edited by Sailing He, Laurent Vivien, Proc. of SPIE Vol. 11284, 112841A · @ 2020 SPIE · CCC code: 0277-786X/20/\$21 · doi: 10.1117/12.2550844 
strong pump beam with wave vector, $\boldsymbol{k}_{\boldsymbol{p}}$ interacts with a weak IR signal field incident under and oblique angle of incidence defined by $\boldsymbol{k}_{i r}$. The resultant upconverted field is produced in a direction, $\boldsymbol{k}_{u p}$ fulling the conservation of energy and momentum respectively ${ }^{3}$. The IR signal can be coherent or incoherent in nature, it is interesting to note that incoherent light, even at the single photon level can be upconverted efficiently ${ }^{4}$. Ideally, the crystal needs to be transparent at all three wavelengths, however this cannot always be achieved. The consequence is an increased noise and reduce conversion efficiency of the upconversion process.

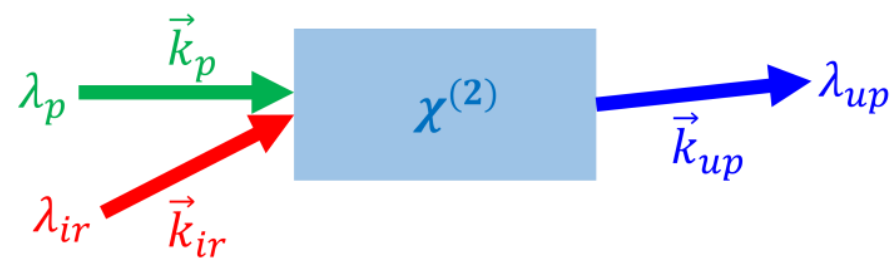

Figure 1. Upconversion imaging using a $\chi^{(2)}$ medium. Three-wave mixing takes place inside the crystal. Upconversion imaging can be considered a generalized version of sum frequency generation with beams interacting under an angle, however characterized by the pump beam being much stronger than the incoming IR signal.

In practice, the mid-IR can be divided into two wavelength regions. A first section, below approximately $5 \mu \mathrm{m}$, where $\mathrm{LiNbO}_{3}(\mathrm{LN})$ or periodically poled LN (PPLN) can be used, see Fig.2. In the majority of applications in this range, the ability to obtain phase matching through periodic inversion of the LN crystal domain offers a versatile solution, providing good conversion efficiency as well as low noise in the upconversion process ${ }^{5}$. A second preferred option may be periodically poled KTP. Note that the transparency range of KTP is significantly smaller as compared to LN.

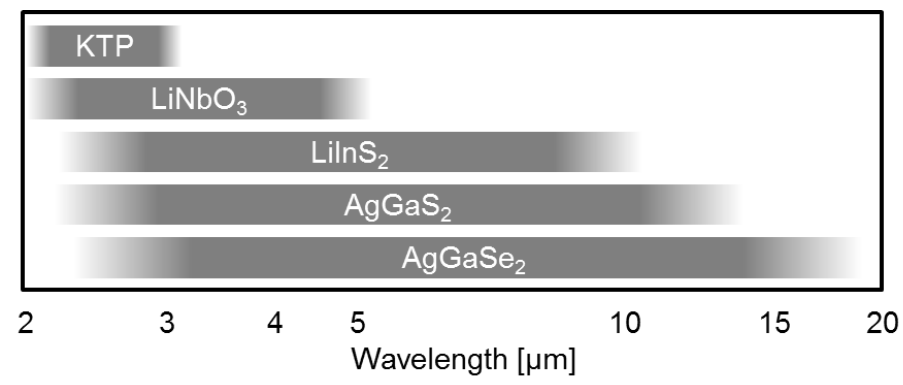

Figure 2. Selected nonlinear crystals used for mid-IR upconversion. PPLN is the preferred solution when below $5 \mu \mathrm{m}$.

The noise properties of upconversion followed by NIR detection is inherently good compared to other options such as direct band gap detectors. In upconversion, the mid-IR incident light is not absorbed and transferred in to an electron in the detector. It is rather translated from the mid-IR to the NIR in a (ideally) transparent medium through the $\chi^{(2)}$ process. Kirchhoff's radiation law states that for a medium in thermal equilibrium, the absorptivity equals the emissivity. Consequently, for a transparent crystal at the IR signal wavelengths, the finite temperature of the crystal will not lead to unwanted Planck radiation, that can be upconverted to the NIR for subsequent detected. This contrasts fundamentally direct mid-IR detectors that requires cryogenic cooling to avoid dark noise, associated with a room temperature environment ${ }^{6}$. $\mathrm{LN}$ is highly transparency below $4 \mu \mathrm{m}$. Above $4 \mu \mathrm{m}$, thermal noise originating from the crystals residual absorption starts to dominate other noise sources, such as upconverted parametric fluorescence.

Above $5 \mu \mathrm{m}$ alternative crystals have to be considered such as $\mathrm{AgGaS}_{2}$ (AGS) or $\mathrm{AgGaSe}_{2}$ (AGSE). While these crystals do have a nonlinearity comparable to PPLN, these crystals suffer from absorption and thermal lensing effects due to poor thermal properties. Adding to this the fact that the optical damage threshold of these crystals are typically smaller, makes these crystals much more demanding to deploy when compared to PPLN. As a consequence, continuous wave (CW), intra-cavity designs, using AGS requires a very carefully designed system ${ }^{7}$. Even then, circulating powers are almost and order of magnitude smaller compared to a well-designed intra-cavity system using PPLN or PPKTP $(>100 \mathrm{~W}$ circulating power). Under pulsed operation, these drawbacks can be partly eliminated. Above $5 \mu \mathrm{m}$ upconversion systems are typically dominated by upconverted thermal noise. Note that Planck radiation peaks at room temperature is approximately at $10 \mu \mathrm{m}$, thus adding thermal noise to the IR signals in this range. 


\subsection{Imaging configuration}

Imaging is typically demonstrated in one of the following three different configurations:

Raster scan imaging: A first obvious implementation comprises a raster scanning system where the object is illuminated point-by-point using e.g. a Quantum Cascade Laser (QCL). In such an implementation, monochromatic images can be obtained where the spatial resolution is determined by the focusing properties of the laser and the lens optics (objective lense). The spectral coverage is determined by the tuning properties of the QCL combined with the angular phase matching properties of the nonlinear crystal. Often a spectral band of several $\mu \mathrm{m}$ can be covered. The advantage of a raster scanning system is several fold. Firstly, an arbitrary large (set by the mechanical scanning stage) object can be scanned. Secondly, collinear interaction in the upconversion process is possible for the full image, which enhances the detection efficiency, and thirdly a high signal-to-noise can typically be obtained, since all laser power is used per point illuminated. The main drawback is the price and additional complexity when using a mechanical scanning stage. Furthermore, the speed is compromised due to the mechanical movement needed. The main requirement of the nonlinear crystal is the angular tuning of the phase matching condition allowing to cover the wavelength range of interest, combined with a high effective nonlinearity to insure good signal-to-noise ratio in the detection ${ }^{8}$.

Polychromatic imaging: If the light source is polychromatic in nature, such as a globar, an object can be imaged with upconversion without tuning the phase match properties. In the following we will refer to a 4-f imaging set-up as described in ref ${ }^{9,10}$. In a 4-f set-up, the nonlinear crystal is positioned in the Fourier plane of the first lens, thus the image information is encoded in angle. Since momentum conservation (phase matching) in birefringent crystals preserves (after size scaling) the image information, the upconverted field containing the image information can be transformed back to spatial coordinates using the second 2-f lens. When upconverting in the Fourier plane, any angle within the FoV will be phase matched at a specific wavelength of the polychromatic light source, thus providing a full FoV. Hence, the image will have a radial wavelength profile depending on the phase matching curve ${ }^{9}$. As an example, a $\pm 5^{\circ}$ FoV (internal crystal angles) for the AGS crystal will contain a bandwidth of approximately $250 \mathrm{~nm}$, while for the case of LIS this will be almost $400 \mathrm{~nm}$, see Fig.3.
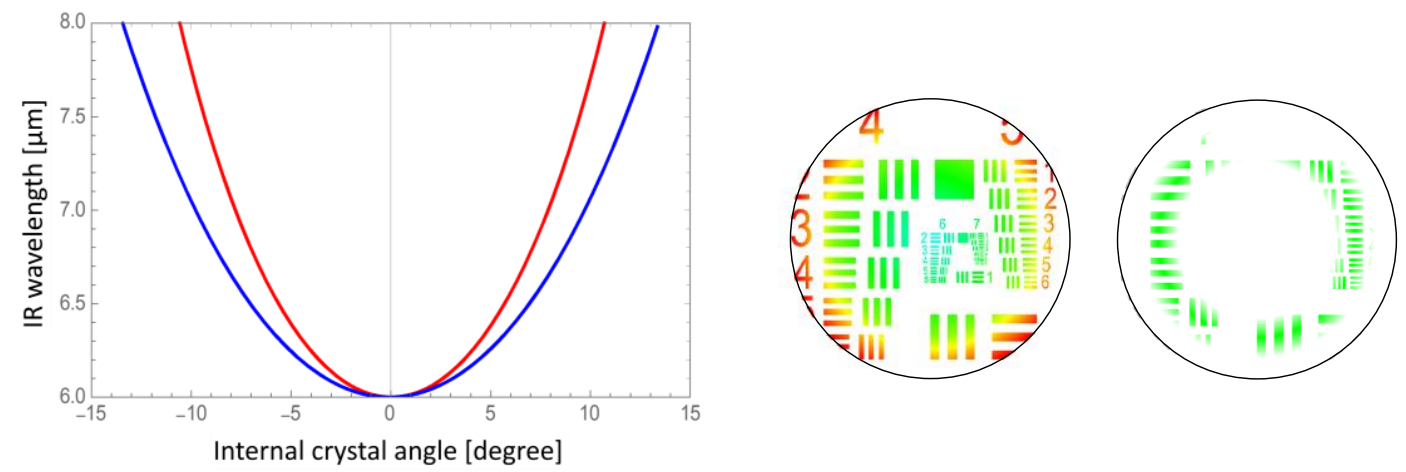

Figure 3. Phase matched IR wavelengths as function of the internal angle of the mid-IR signal relative to the pump. The blue (AGS) and red (LIS) graphs show the phase matched IR wavelengths when upconverting with a $1064 \mathrm{~nm}$ pump source. Right hand figures show upconverted polychromatic and monochromatic images, respectively.

Monochromatic imaging: In case of monochromatic illumination, only a smaller FoV is upconverted, limited by the monochromatic phase matching as seen in Fig. 3. If larger images are wanted, scanning of the phase matching is required, which correspond to shifting the phase match graphs up/down, while keeping the other parameters fixed. This can be obtained e.g. by rotation of the nonlinear material, i.e. change the angle of the three interacting fields relative to the optical axis of the material. In ref $^{11}$, the object was spatially scanned by rotating the nonlinear crystal, upconverting individual concentric ring shaped sections of the object at a time. The phase matching curve possess close to rotational symmetry around the wavelength axis, see Fig. 3. This provides a mechanism for obtaining large FoV upconverted images, by scanning the angle of the nonlinear material, while adding the individual frames. From a design point of view, the less steep the parabola, the better, as this offers fatter donut shaped regions for fixed crystal angles. Often, only a few degrees of crystal rotation is necessary to increase the FoV significantly, thus making the imaging system fast and reliable. 


\subsection{Upconversion spectrometer}

The radial wavelength sweep discussed in section 2.2 can be used as a spectrometer by analysing the radial intensity profile of the upconverted light. A spectral resolution in the order of $8 \mathrm{~cm}^{-1}$ was demonstrated in ref ${ }^{12}$ at approximately 9 $\mu \mathrm{m}$ using AGS, where the phase matched acceptance bandwidth limit the resolution. In order to increase the spectral coverage, a larger cone of angles is needed in the polychromatic illumination. Here a shallow parabola is preferred as this limits the angles needed to cover a desired wavelength range, see Fig. 3.

It is possible to decouple the crystal acceptance parameters from the spectral resolution. In ref $^{13}$ a grating system was inserted as a part of a 2-f set-up. The upconverted light emerges from a virtual pinhole formed by the overlap of the focused pump laser beam and the incident mid-IR light inside the nonlinear crystal. Consequently, the upconverted light can be collimated onto a grating, dispersing the light, and focusing onto a CCD camera system or a line array as in a normal spectrometer.

\subsection{Filtering}

For a standard mid-IR detector, the incident optical signal is absorbed in the detector material and converted into an electrical signal, see Fig. 4(a). Mid-IR upconversion offers an additional option for filtering, since the upconverted (NIR) signal is still in the optical regime. As indicated in Fig. 4(b) both spectral and spatial optical filters can be inserted between the upconversion section and the NIR detector. In situations where stray light sources are known, or where the upconverted signal is expected in a narrow wavelength band several orders of magnitude improvement in signal-to-noise can be obtained.

(a)

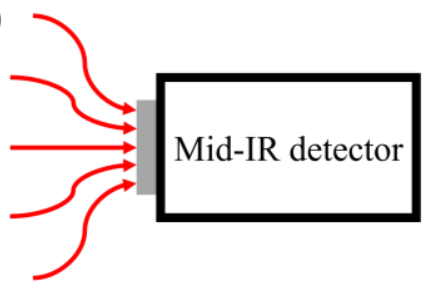

(b)

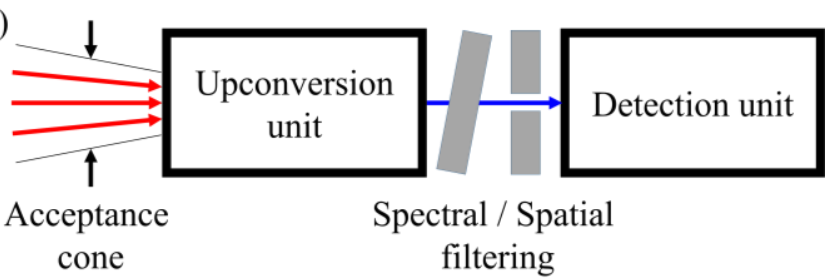

Figure 4. (a) Direct mid-IR detection versus (b) upconversion detection. Red color represents the IR field and blue color represents the upconverted field.

A second feature improving signal-to-noise is the acceptance angle imposed by the upconversion process, see Fig. 4(b). Often the acceptance angle/cone is only a few degrees, thus preventing stray light at large angles of incidence to be upconverted efficiently. The small acceptance angle is obviously a disadvantage in cases where a large etendue is needed.

\subsection{Short pulse upconversion}

In this section, properties of an upconversion imaging system is briefly discussed in terms of the temporal aspects of the interacting fields. Acceptance parameters as well as efficiency of an upconversion imaging process is influenced by the length of the nonlinear crystal with increased crystal length leading to increase in nonlinear conversion efficiency while decrease in angular and spectral acceptance bandwidth ${ }^{3}$. Angular and spectral acceptance bandwidth dictates the FoV while imaging. From $\mathrm{CW}$ up to few picosecond pulse regime, the efficiency and acceptance parameters remain fixed for the system. For short pulses (below few picoseconds), the temporal overlap between the interacting field will decrease due to group velocity mismatch (GVM) between the interaction pulses. As a result, the field interaction length (or the 
effective length of the crystal) is smaller than the actual length of the crystal, this phenomena is not encountered in long pulse or CW upconversion systems. A reduction in effective length of the crystal drastically reduces the monochromatic collinear plane wave efficiency but increases the acceptance parameters for a short pulse system. A detailed discussion on a femtosecond upconversion imaging system can be found in ref $^{14}$. For the system described in ref ${ }^{14}$, an angular acceptance bandwidth as high as $20^{\circ}$ and a spectral acceptance bandwidth as high as $147 \mathrm{~nm}$ is estimated. In contrast, the acceptance parameter of a similar system in the long pulse/CW regime would comparatively be very small (refer to Table 1 in ref $^{14}$ ). The increased FoV due to the increase in angular and spectral acceptance enables single shot upconversion imaging without (or with limited) the need to tune the phase matching condition. As we move to shorter pulse durations for the interacting fields, the effective interaction length becomes shorter. It then becomes practical to use a shorter crystal having a length equal to the GVM limited interaction length, which also brings down the cost associated with the system.

\section{CONCLUSIONS}

Upconversion imaging and detection is a viable alternative to direct detection in many mid-IR configurations. The nonlinear crystals transparency range is a central parameter, since even modest absorption at the mid-IR wavelength will result in increased noise in the upconversion process. The phase matching properties of the crystal is vital for upconversion of spatial properties of the mid-IR objects. AGS and LIS was compared for upconversion imaging and spectroscopy respectively.

Using the possibility to spectrally and spatially filter at the upconverted wavelengths is vital for low noise performance, often providing orders of magnitude improvement in signal-to-noise ratio compared to direct detection.

$\mathrm{CW}$ wave theory is adequate down to the few ps regime, however group velocity mismatch between the three interacting waves inside the crystal will eventually lead to a decrease in efficiency. As the effective interaction length decreases, acceptance parameters increases correspondingly increasing the acceptance angles. In pulsed operation synchromous upconversion is preferred, since high efficiency can be maintained.

\section{REFERENCES}

[1] J. M. Chalmers and P. R. Griffiths, [Handbook of vibrational spectroscopy], Wiley Publishers, Chichester (2002)

[2] M. Ebrahim-Zadeh and I. T. Sorokina, [Mid-infrared coherent sources and applications], Springer Publishers, Dordrecht (2008)

[3] B. E. A. Saleh and M. C. Teich, [Fundamentals of photonics], Wiley Publishers, Chichester (2013)

[4] J. S. Dam, P. Tidemand-Lichtenberg, and C. Pedersen, "Room-temperature mid-infrared single-photon spectral imaging," Nature Photonics 6(11), 788-793 (2012)

[5] L. Høgstedt, J. S. Dam, A-L. Sahlberg, Z. Li, M. Aldén, C. Pedersen, and P. Tidemand-Lichtenberg., "Low-noise mid-IR upconversion detector for improved IR-degenerate four-wave mixing gas sensing," Opt. Lett. 39(18), 5321, (2014)

[6] A. Rogalski, [Infrared Detectors], CRC Press (2010)

[7] S. Junaid, P. Tidemand-Lichtenberg, and C. Pedersen, "Upconversion based spectral imaging in 6 to $8 \mu \mathrm{m}$ spectral regime," Proc. SPIE 10088 (2017)

[8] Y-P. Tseng, P. Bouzy, C. Pedersen, N. Stone, and P. Tidemand-Lichtenberg, "Upconversion raster scanning microscope for long-wavelength infrared imaging of breast cancer microcalcifications," Biomed. Opt. Express 9(10), 4979 (2018)

[9] P. Tidemand-Lichtenberg, J. S. Dam, H. V. Andersen, L. Høgstedt, and C. Pedersen, "Mid-infrared upconversion spectroscopy," J. Opt. Soc. Am. B 33(11), D28 (2016)

[10] H. Maestre, A. J. Torregrosa, and J. Capmany, "IR Image upconversion using band-limited ASE illumination fiber sources," Opt. Express 24(8), 8581 (2016) 
[11] S. Junaid, S. Chaitanya Kumar, M. Mathez, M. Hermes, N. Stone, N. Shepherd, M. Ebrahim-Zadeh, P. TidemandLichtenberg, and C. Pedersen, "Video-rate, mid-infrared hyperspectral upconversion imaging," Optica 6(6), 702 (2019)

[12] S. Junaid, J. Tomko, M. P. Semtsiv, J. Kischkat, W. T. Masselink, C. Pedersen, and P. Tidemand-Lichtenberg, "Mid-infrared upconversion based hyperspectral imaging," Opt. Express 26(3), 2203 (2018)

[13] S. Wolf, J. Kiessling, M. Kunz, K. Buse, and F. Kühnemann, "Upconversion-enabled array spectrometer for the mid-infrared, featuring kilohertz spectra acquisition rates," Opt. Express 25(13), 12 (2017)

[14] Ashik. A. S., C. F. O’Donnell, S. Chaitanya Kumar, M. Ebrahim-Zadeh, P. Tidemand-Lichtenberg, and C. Pedersen, "Mid-infrared upconversion imaging using femtosecond pulses," Photon. Res. 7(7), 783 (2019) 